Pacific

Journal of

Mathematics

TIGHT CONTACT STRUCTURES OF CERTAIN SEIFERT FIBERED 3-MANIFOLDS WITH $e_{0}=-1$

JINHONG KIM 


\title{
TIGHT CONTACT STRUCTURES OF CERTAIN SEIFERT FIBERED 3-MANIFOLDS WITH $e_{0}=-1$
}

\author{
JINHONG KIM
}

\begin{abstract}
We classify, up to contact isotopy, all tight contact structures on a family of Seifert fibered three-manifolds $M\left(-\frac{1}{2}, \frac{1}{3}, \frac{\beta}{\alpha}\right)$ satisfying $0<\frac{\beta}{\alpha}<\frac{1}{6}$. We show that, if $\left[r_{0}, r_{1}, \ldots, r_{l}\right]$ is the continued fraction expansion of $-\frac{\alpha}{\beta}$, there are exactly $\left|r_{0}+5\right|\left|r_{1}+1\right| \cdots\left|r_{l}+1\right|$ tight contact structures on such Seifert fibered three-manifolds $M\left(-\frac{1}{2}, \frac{1}{3}, \frac{\beta}{\alpha}\right)$ as above, so all the tight contact structures are holomorphically fillable.
\end{abstract}

\section{Introduction and results}

An oriented 2-plane field distribution $\xi$ on an oriented 3-manifold is called a positive contact structure if $\xi=\operatorname{ker} \eta$ for some global 1-form $\eta$ satisfying $\eta \wedge d \eta>0$. In this paper, a contact structure always means a positive contact structure. All contact structures in 3-manifolds fall largely into two categories: tight and overtwisted (see [Honda 2000] for details).

Let $M\left(r_{1}, r_{2}, r_{3}\right)$ denote a (small) Seifert fibered 3-manifold over $S^{2}$ with three singular fibers. The integer

$$
e_{0}\left(M\left(r_{1}, r_{2}, r_{3}\right)\right)=\sum_{i=1}^{3}\left[r_{i}\right]
$$

is an invariant of the Seifert fibered 3-manifold $M\left(r_{1}, r_{2}, r_{3}\right)$, called the truncated Euler number. H. Wu [2004] has classified tight contact structures on small Seifert fibered 3-manifolds with $e_{0} \neq-2,-1,0$; subsequently P. Ghiggini, P. Lisca, and A. Stipsicz extended the classification results of $\mathrm{Wu}$ to the case $e_{0}=0$ [Ghiggini et al. 2004].

Here we report the classification of tight contact structures, up to contact isotopy, on a family of the Seifert fibered 3-manifolds $M\left(-\frac{1}{2}, \frac{1}{3}, \frac{\beta}{\alpha}\right)$ satisfying $0<\frac{\beta}{\alpha}<\frac{1}{6}$. Note that the Seifert fibered 3-manifolds of the present paper have the truncated Euler number $e_{0}=-1$ which was considered as kind of an exceptional case in [Wu 2004] and [Ghiggini et al. 2004].

MSC2000: 57R57.

Keywords: tight contact structure, Seifert fibered 3-manifold, truncated Euler number.

This work was supported by Korea Research Foundation Grant KRF-2004-041-C00048. 
To state the main result, we write $-\frac{\alpha}{\beta}$ as a continued fraction $\left[r_{0}, r_{1}, \ldots, r_{l}\right]$.

Theorem 1.1. Let $M$ be the Seifert fibered 3-manifold $M\left(-\frac{1}{2}, \frac{1}{3}, \frac{\beta}{\alpha}\right)$ with $0<\frac{\beta}{\alpha}<$ $\frac{1}{6}$. Then there exist exactly $\left|r_{0}+5\right|\left|r_{1}+1\right| \cdots\left|r_{l}+1\right|$ tight contact structures on $M$ up to contact isotopy. So all the tight contact structures on $M$ are holomorphically fillable.

As an immediate consequence, we have the following

Corollary 1.2. On the Seifert fibered 3-manifold $M\left(-\frac{1}{2}, \frac{1}{3}, \frac{2}{6 k-1}\right)$ with $k \geq 2$, there exist exactly $3 k-5$ tight contact structures up to contact isotopy. So all the tight contact structures on $M\left(-\frac{1}{2}, \frac{1}{3}, \frac{2}{6 k-1}\right)$ with $k \geq 2$ are holomorphically fillable.

This corollary answers a question of P. Ghiggini and S. Schönenberger [2003, Section 5]. Note also that $M\left(-\frac{1}{2}, \frac{1}{3}, \frac{2}{11}\right)$ is just the Brieskorn homology 3-sphere $-\Sigma(2,3,11)$ equipped with orientation opposite to the one as a boundary of the Milnor fiber. The special case of Theorem 1.1 for $k=2$ is proved in [Ghiggini and Schönenberger 2003]. It would also appear to be interesting to classify tight contact structures on the Brieskorn homology 3 -spheres $-\Sigma(2,3,6 k-1)(k>2)$ equipped with orientation opposite to the one as a boundary of the Milnor fiber. Lisca and Matić [1997] showed that there exist at least $k-1$ tight contact structures on $-\Sigma(2,3,6 k-1)$ which are holomorphically fillable. A preliminary analysis analogous to the present paper shows that there seem to exist exactly $\frac{k(k-1)}{2}$ tight contact structures on the Brieskorn homology 3 -sphere $-\Sigma(2,3,6 k-1)$; this work will appear elsewhere. So the result of Theorem 1.1 seems to be sharp in the sense that $-\Sigma(2,3,6 k-1)$ is the Seifert 3-manifold $M\left(-\frac{1}{2}, \frac{1}{3}, \frac{k}{6 k-1}\right)$ in our notation and that $\frac{k}{6 k-1}$ is greater than $\frac{1}{6}$. We hope to come back to the classification of tight contact structures on $-\Sigma(2,3,6 k-1)$ in the future work.

As a consequence of Theorem 1.1, each Seifert fibered 3-manifold $M\left(-\frac{1}{2}, \frac{1}{3}, \frac{\beta}{\alpha}\right)$ has exactly $\left|r_{0}+5\right|\left|r_{1}+1\right| \cdots\left|r_{l}+1\right|$ nonisotopic tight (in fact, holomorphically fillable) contact structures satisfying $0<\frac{\beta}{\alpha}<\frac{1}{6}$. Hence these same manifolds are atoroidal (have no incompressible tori); this follows from a recent theorem of Colin, Giroux, and Honda [Colin et al. 2003, Theorem 0.5], to the effect that a closed, oriented, irreducible 3-manifold carries infinitely many tight contact structures up to isotopy if and only if it is toroidal.

We prove Theorem 1.1 by essentially employing the techniques developed in [Honda 2000; Etnyre and Honda 2001] and later implemented in [Ghiggini and Schönenberger 2003]. This paper is a result of the author's attempt to understand those techniques. We use the same definitions, terminology, and theorems as in the three references just cited. The reader is expected to be familiar with those papers.

Much of this article is devoted to the classification results of the Seifert fibered 3-manifold $M\left(-\frac{1}{2}, \frac{1}{3}, \frac{2}{6 k-1}\right)$ for $k \geq 3$, in detail; we think that the analysis of 
these special cases will help the reader understand the proof of the general case. Specifically, in Section 2 we give an upper bound of the number of Corollary 1.2, and in Section 3 we construct $3 k-5$ holomorphically fillable contact structures using the Legendrian surgery description as in [Gompf 1991; 1998; [Eliashberg 1990]]. Section 4 gives a proof of the general case of a Seifert fibered 3-manifold $M\left(-\frac{1}{2}, \frac{1}{3}, \frac{\beta}{\alpha}\right)$ satisfying the property $0<\frac{\beta}{\alpha}<\frac{1}{6}$.

\section{Proof of Corollary 1.2: upper bound}

In this section $M$ denotes the Seifert fibered 3-manifold $M\left(-\frac{1}{2}, \frac{1}{3}, \frac{2}{6 k-1}\right)$ over $S^{2}$ with three singular fibers $f_{i}$ with invariants $\left(-\frac{1}{2}, \frac{1}{3}, \frac{2}{6 k-1}\right)$. Let $V_{i}(i=1,2,3)$ be tubular neighborhoods of the singular fibers $f_{i}$, and we identify $\partial V_{i}$ with $\mathbb{R}^{2} / \mathbb{Z}^{2}$ by choosing $(1,0)^{T}$ as the meridional direction and $(0,1)^{T}$ as a longitudinal direction. Let $\Sigma$ denote a pair of pants. We identify each component of $-\partial\left(S^{1} \times \Sigma\right)$ with $\mathbb{R}^{2} / \mathbb{Z}^{2}$ in a similar way as above. Then $M\left(-\frac{1}{2}, \frac{1}{3}, \frac{2}{6 k-1}\right)$ can be obtained by attaching the solid tori $V_{i}$ to $S^{1} \times \Sigma$ via the attaching maps

$$
A_{i}: \partial V_{i} \rightarrow-\partial\left(S^{1} \times \Sigma\right)
$$

defined by

$$
A_{1}=\left(\begin{array}{rr}
2 & -1 \\
1 & 0
\end{array}\right), \quad A_{2}=\left(\begin{array}{rr}
3 & 1 \\
-1 & 0
\end{array}\right), \quad A_{3}=\left(\begin{array}{cc}
6 k-1 & 3 k \\
-2 & -1
\end{array}\right) .
$$

Now we recall the definition of the twisting number of a Legendrian curve used in this paper. A Legendrian knot in the manifold $M$ which is smoothly isotopic to a regular fiber admits two framings, the fibration framing and the contact framing. The difference between the contact framing and the fibration framing is called the twisting number of the Legendrian curve.

Theorem 2.1. On the Seifert fibered 3-manifold $M\left(-\frac{1}{2}, \frac{1}{3}, \frac{2}{6 k-1}\right)$ with $k \geq 3$, there exist, up to contact isotopy, at most $3 k-5$ tight contact structures.

Proof. The proof of the theorem consists of several steps. We first assume without loss of generality that the singular fibers $f_{i}$ are simultaneously isotoped to Legendrian curves with negative twisting numbers $n_{i}$ for $i=1,2,3$. Then the slopes of $\partial V_{i}$ of a standard neighborhood $V_{i}$ of $f_{i}$ with convex boundary become $1 / n_{i}$. Furthermore, we assume that the Legendrian ruling slope on $-\partial\left(M \backslash V_{i}\right)$ is infinite, due to the flexibility of the Legendrian rulings [Honda 2000, Theorem 3.4; Ghiggini and Schönenberger 2003, Theorem 3].

Lemma 2.2. We can increase the twisting numbers $n_{1}$ and $n_{2}$ up to -2 , and the twisting number $n_{3}$ up to -1 . 
Proof. By assumption, the boundary slopes of $-\partial\left(M \backslash V_{i}\right)$ under $A_{i}$ are

$$
\begin{gathered}
s\left(-\partial\left(M \backslash V_{1}\right)\right)=\frac{n_{1}}{2 n_{1}-1}, \quad s\left(-\partial\left(M \backslash V_{2}\right)\right)=\frac{-n_{2}}{3 n_{2}+1}, \\
s\left(-\partial\left(M \backslash V_{3}\right)\right)=-\frac{2 n_{3}+1}{(6 k-1) n_{3}+3 k} .
\end{gathered}
$$

Let $A$ be a convex annulus whose boundary consists of Legendrian rulings of $-\partial\left(M \backslash V_{1}\right)$ and $-\partial\left(M \backslash V_{2}\right)$. We have three cases to consider:

Case 1. $2 n_{1}-1 \neq 3 n_{2}+1$. Assume first that $2 n_{1}-1<3 n_{2}+1 \leq 0$. Then it follows from the Imbalance Principle [Honda 2000, Proposition 3.17] that there exists a bypass along a Legendrian ruling on $-\partial\left(M \backslash V_{1}\right)$. By the Twisting Number Lemma [Honda 2000, Lemma 4.4] with Legendrian ruling slope $r_{1}=2$, we can increase the twisting number $n_{1}$ by 1 as long as $n_{1} \leq-1$. Next, if $3 n_{2}+1<2 n_{1}-1 \leq 0$, then similarly with $r_{2}=-\frac{1}{3}$ we can increase the twisting number $n_{2}$ by 1 as long as $n_{2} \leq-2$.

To sum up, we can increase the twisting numbers $n_{1}$ and $n_{2}$ by one at least as long as $n_{1} \leq-1$ and $n_{2} \leq-2$.

Case 2. $2 n_{1}-1=3 n_{2}+1$ and there exists a bypass on the convex annulus. In this case we apply the Twisting Number Lemma as in Case 1 to increase the twisting numbers.

Case 3. $2 n_{1}-1=3 n_{2}+1$ and there exists no bypass on the convex annulus. In this case we cannot apply the Twisting Number Lemma. So we apply the cut-andround procedure [Ghiggini and Schönenberger 2003, Proposition 6; Honda 2000, Lemma 3.11] along a convex annulus $A$ to get a convex torus isotopic to $\partial\left(M \backslash V_{3}\right)$ with boundary slope

$$
\frac{n_{1}}{2 n_{1}-1}-\frac{n_{2}}{3 n_{2}+1}-\frac{1}{2 n_{1}-1}=\frac{n_{2}}{6 n_{2}+2}=\frac{p}{6 p+1},
$$

where $n_{2}=2 p$ for $p \leq-1$. Since

$$
\left(\begin{array}{rc}
-1 & -3 k \\
2 & 6 k-1
\end{array}\right)\left(\begin{array}{c}
6 n_{2}+2 \\
-n_{2}
\end{array}\right)=\left(\begin{array}{c}
3(k-2) n_{2}-2 \\
-(6 k-13) n_{2}+4
\end{array}\right),
$$

we obtain a boundary slope $-2+n_{2} /\left(3(k-2) n_{2}-2\right)$ on $\partial V_{3}$, which is negative.

We now work with a convex annulus $A$ between $-\partial\left(M \backslash V_{2}\right)$ and $-\partial\left(M \backslash V_{3}\right)$. Since $6 n_{2}+2<3 n_{2}+1 \leq 0$, we can apply the Imbalance Principle to $A$ to obtain a bypass on $\partial V_{3}$. Then by the Twisting Number Lemma with the Legendrian ruling slope

$$
r_{3}=\frac{-(6 k-13) n_{2}+4}{(3 k-6) n_{2}-2}
$$


we can increase the twisting number $n_{3}$ by 1 as long as $n_{3} \leq-2$. In fact, since

$$
-2 \leq-1+\frac{1}{r_{3}}=\frac{(3 k-6) n_{2}-2}{-(6 k-13) n_{2}+4} \leq-\frac{3}{2}\left(1+\frac{1}{18 k-27}\right)<-\frac{3}{2},
$$

we should have

$$
n_{3} \leq-1+\frac{1}{r_{3}}
$$

as long as $n_{3} \leq-2$. This implies that we can increase the twisting number $n_{3}$ up to -1 .

Now in order to increase the twisting numbers $n_{1}$ and $n_{2}$, notice that as above the possible boundary slopes on $\partial\left(M \backslash V_{3}\right)$ are $p /(6 p+1)$ for $p \leq-1$. Since $2 /(6 k-1)<\frac{1}{6}<p /(6 p+1)$ for $k \geq 3$, inside $V_{3}$ there must exist a standard torus $T$ with boundary slope $\frac{1}{6}$. Now, if we compare a Legendrian curve on $T$ isotopic to the regular fiber with another Legendrian curve isotopic to the regular fiber on $\partial\left(M \backslash V_{3}\right)$, then clearly we obtain a bypass for $\partial\left(M \backslash V_{3}\right)$ except when $p /(6 p+1)=\frac{1}{5}$ (i.e., $\left.p=-1\right)$. Thus we can increase the twisting number $n_{2}$ (and so $\left.n_{1}\right)$ up to -2 .

From these three cases, we see that the twisting numbers $n_{1}, n_{2}$, and $n_{3}$ can be increased up to $-2,-2$, and -1 , respectively. This completes the proof.

Remark 2.3. According to the referee, there exists a much simpler argument to deal with Case 3 in the proof of Lemma 2.2 as follows. Namely, let $T^{\prime}$ be the convex torus isotopic to $\partial\left(M \backslash V_{3}\right)$ obtained by the cut-and-round procedure with boundary slope $p /(6 p+1)$, and let $T$ be the convex torus with boundary slope $\frac{1}{6}$ $(<p /(6 p+1))$ as above. Then take a vertical annulus from $T$ to $T^{\prime}$ and use the Imbalance Principle to obtain a bypass for $T$ along a ruling curve of slope $\infty$. This then can be used to thicken $V_{1}$ and $V_{2}$.

From now on, we assume that the twisting numbers $n_{i}$ are $n_{1}=n_{2}=-2$ and $n_{3}=-1$. Let $A$ denote a convex vertical annulus whose boundary consists of Legendrian rulings of $\partial\left(M \backslash V_{1}\right)$ and $\partial\left(M \backslash V_{2}\right)$. Then we divide our proof into two cases, depending on whether or not $A$ has a boundary-parallel dividing curve.

Lemma 2.4. Assume that $A$ has a boundary-parallel dividing curve. Then we can further increase the twisting numbers $n_{1}, n_{2}$, and $n_{3}$ up to $0,-1$, and -1 , respectively. Furthermore, there exists a regular fiber with twisting number 0.

Proof. By taking the configurations of dividing curves on the annulus $A$ into account, we see that there must exist a bypass on each side of $-\partial\left(M \backslash V_{1}\right)$ and $-\partial\left(M \backslash V_{2}\right)$. By the Twisting Number Lemma, we can increase $n_{1}$ and $n_{2}$ up to -1 . But then there must be one more bypass on $-\partial\left(M \backslash V_{1}\right)$ by the Imbalance principle, since $t\left(-\partial\left(M \backslash V_{1}\right)\right)=-3<t\left(-\partial\left(M \backslash V_{2}\right)\right)=-2<0$. Hence we can increase the 
twisting number $n_{1}$ up to 0 . With these new twisting numbers $n_{1}=0$ and $n_{2}=-1$, we have the boundary slopes $s\left(-\partial\left(M \backslash V_{1}\right)\right)=0$ and $s\left(-\partial\left(M \backslash V_{2}\right)\right)=-\frac{1}{2}$.

Now we apply the cut-and-round procedure to the convex annulus $A$. Then we obtain a convex torus with boundary slope $-\frac{1}{2}+0+\frac{1}{2}=0$ on a torus isotopic to $\partial\left(M \backslash V_{3}\right)$. This implies that the slope of $\partial V_{3}$ can be assumed to be -2 . Hence by Proposition 18 in [Ghiggini and Schönenberger 2003] we can find a convex torus parallel to the boundary $\partial V_{3}$ with boundary slope $-\frac{6 k-1}{3 k}$, since $-2<-2+\frac{1}{3 k}=$ $-\frac{6 k-1}{3 k}<0$. But this slope is equivalent to infinity on $-\partial\left(M \backslash V_{3}\right)$. Hence we have a regular fiber with twisting number 0 . This completes the proof.

Proposition 2.5. Under the assumptions of Lemma 2.4, there does not exist, up to contact isotopy, any tight contact structure on $M\left(-\frac{1}{2}, \frac{1}{3}, \frac{2}{6 k-1}\right)$ having twisting numbers $n_{1}=0, n_{2}=-1$, and $n_{3}=-1$.

Proof. We use a slight modification of the proof in [Ghiggini and Schönenberger 2003, Section 4.2.2]. For the reader's sake, however, we give it here in some detail.

By Lemma 2.4, $M \backslash \bigcup_{i=1}^{3} V_{i}$ is diffeomorphic to $S^{1} \times \Sigma$ with boundary slopes $0,-\frac{1}{2}$, and $-\frac{1}{3 k-1}$, and further there exists another pair of pants $\Sigma^{\prime}$ inside $\Sigma$ so that $S^{1} \times \Sigma^{\prime}$ is diffeomorphic to $M \backslash \bigcup_{i=1}^{3} V_{i}^{\prime}$ with all boundary slopes equal to $\infty$, where $V_{i}^{\prime}$ is a neighborhood of the singular fiber $f_{i}$ containing $V_{i}$ for $i=1,2,3$. Hence $S^{1} \times\left(\Sigma-\Sigma^{\prime}\right)$ is diffeomorphic to the union of three thickened tori $T_{i} \times I$ so that $T_{i} \times\{0\}=-\partial\left(M \backslash V_{i}\right)$ and $T_{i} \times\{1\}=-\partial\left(M \backslash V_{i}^{\prime}\right)$.

Since $T_{1} \times I$ has boundary slopes 0 and $\infty$, and $\frac{0}{1}$ and $\frac{1}{0}$ are consecutive in the Farey tessellation, $T_{1} \times I$ is a basic slice. Similarly, since $-\frac{1}{2},-1$, and $\frac{1}{0}$ are consecutive, $T_{2} \times I$ is the union of two basic slices $T_{2} \times\left[0, \frac{1}{2}\right]$ with boundary slopes $-\frac{1}{2}$ and -1 and $T_{2} \times\left[\frac{1}{2}, 1\right]$ with boundary slopes -1 and $\infty$, and $T_{3} \times I$ with boundary slopes $-\frac{1}{3 k-1}$ and $\infty$ is the union of $3 k-1$ basic slices $T_{3} \times\left[\frac{i}{3 k-1}, \frac{i+1}{3 k-1}\right]$ with boundary slopes $-1 /(3 k-1-i)$ and $-1 /(3 k-2-i)$ for $i=0,1, \ldots, 3 k-2$.

Let $p_{i}$ denote the number of positive basic slices in $T_{i} \times I$. Then we have the following possibilities; $p_{1}=0,1, p_{2}=0,1,2$, and $p_{3}=0,1, \ldots, 3 k-1$. (As the referee pointed out, we can let $p_{3}$ be the number of positive basic slices from slope $-\frac{1}{6}$ to $\infty$. Then $p_{3}=0,1, \ldots, 6$. So we can simplify the argument below. See also Section 4.) Thus there exist at most $2 \times 3 \times 3 k=18 k$ possible tight contact structures on $M \backslash \bigcup_{i=1}^{3} V_{i}$ by Lemma 31 in [Ghiggini and Schönenberger 2003].

First, we need the following definition:

Definition 2.6. Let $\tilde{V}_{i}$ be thickenings of the $V_{i}$ so that the boundary slopes are zero. Their equivalent slopes

$$
s\left(-\partial\left(M \backslash \tilde{V}_{1}\right)\right)=\frac{1}{2}, \quad s\left(-\partial\left(M \backslash \tilde{V}_{2}\right)\right)=-\frac{1}{3}, \quad \text { and } s\left(-\partial\left(M \backslash \tilde{V}_{3}\right)\right)=-\frac{2}{6 k-1}
$$

are called critical. 
In fact, they are slopes for which we can find an overtwisted contact structure on the 3-manifold $M$.

In order to prove the proposition, we need to consider following four cases;

Case 1. We first assume that $T_{3} \times\left[\frac{3 k-3}{3 k-1}, 1\right]$ is contact isomorphic to $T_{2} \times[0,1]$. Note that this assumption covers $p_{2}=0,1$, or 2 . Then the manifold $M \backslash\left(V_{1}^{\prime} \cup\right.$ $\left.V_{2} \cup V_{3} \cup T_{3} \times\left[0, \frac{3 k-3}{3 k-1}\right]\right)$ has boundary slopes $\infty,-\frac{1}{2}$, and $-\frac{1}{2}$. Then it follows from Lemma 36 in [Ghiggini and Schönenberger 2003] that we can find a vertical annulus $A$ between $T_{2} \times\{0\}$ and $T_{3} \times\left\{\frac{3 k-3}{3 k-1}\right\}$ without boundary parallel dividing arcs. Now apply the cut-and-round procedure to the annulus $A$ so that we get a slope $-\frac{1}{2}-\frac{1}{2}+\frac{1}{2}=-\frac{1}{2}$ on a torus isotopic to $\partial\left(M \backslash V_{1}\right)$. But it is equivalent to the slope $\frac{1}{2}$ on a torus isotopic to $-\partial\left(M \backslash V_{1}\right)$ which is critical. Hence these cases do not occur. Thus we are left with the following cases $p_{2}=0$ and $p_{3}=3 k-2$ or $3 k-1, p_{2}=1$ and $p_{3}=0$ or $3 k-1$, or $p_{2}=2$ and $p_{3}=0$ or 1 .

Case 2. Next assume that three basic slices in $T_{3} \times\left[\frac{3 k-4}{3 k-1}, 1\right]$ have the same sign as $T_{1} \times I$. Let $V_{1}^{\prime \prime}$ be a standard neighborhood whose boundary slope $s\left(-\partial\left(M \backslash V_{1}^{\prime \prime}\right)\right)=$ $T_{1} \times\{-1\}$ is $\frac{1}{3}$. Then the manifold

$$
M \backslash\left(V_{1}^{\prime \prime} \cup V_{2}^{\prime} \cup V_{3} \cup T_{3} \times\left[0, \frac{3 k-4}{3 k-1}\right]\right)
$$

has boundary slopes $\frac{1}{3},-\frac{1}{3}$, and $\infty$. Then, by [Ghiggini and Schönenberger 2003, Lemma 37], we can find a vertical annulus $A$ between $T_{1} \times\{-1\}$ and $T_{3} \times\left\{\frac{3 k-4}{3 k-1}\right\}$ without boundary-parallel arcs. Now apply the cut-and-round procedure along $A$ to get the boundary slope $\frac{1}{3}-\frac{1}{3}+\frac{1}{3}=\frac{1}{3}$ on a torus isotopic to $\partial\left(M \backslash V_{2}\right)$. Thus we have a critical boundary $-\frac{1}{3}$ on $-\partial\left(M \backslash V_{2}\right)$. Thus we are left with the following cases $p_{1}=0$ and $p_{3}=3 k-3,3 k-2$, or $3 k-1$ or $p_{1}=1$ and $p_{3}=0,1$, or 2 .

Summarizing the two preceding cases, we are left with six possibilities: $p_{1}=$ $0, p_{2}=0$, and $p_{3}=3 k-2$ or $3 k-1, p_{1}=0, p_{2}=1$ and $p_{3}=3 k-1$ or $p_{1}=1, p_{2}=1$ and $p_{3}=0$ or $p_{1}=1, p_{2}=2$ and $p_{3}=0$ or 1 .

Case 3. This time we assume that the basic slices $T_{2} \times I$ and $T_{1} \times I$ have the same sign. This covers $p_{1}=0, p_{2}=0$ and $p_{1}=1, p_{2}=2$. Since we can decrease the twisting numbers $n_{1}$ and $n_{2}$ up to -2 , we can take standard neighborhood $V_{1}^{\prime \prime}$ and $V_{2}^{\prime \prime}$ for which the boundary slopes are $s\left(-\partial\left(M \backslash V_{1}^{\prime \prime}\right)\right)=\frac{2}{5}$ and $s\left(-\partial\left(M \backslash V_{2}^{\prime \prime}\right)\right)=$ $-\frac{2}{5}$. Thus the manifold $M \backslash\left(V_{1}^{\prime \prime} \cup V_{2}^{\prime \prime} \cup V_{3}^{\prime}\right)$ has boundary slopes $\frac{2}{5},-\frac{2}{5}, \infty$. A similar argument as above yields a convex torus isotopic to $-\partial\left(M \backslash V_{3}\right)$ with slope $-\frac{1}{5}$. The thickened torus between $-\partial\left(M \backslash V_{3}\right)$ and $-\partial\left(M \backslash V_{3}^{\prime \prime}\right)$ has boundary slopes $-\frac{1}{3 k-1}$ and $-\frac{1}{5}$. Since the thickened torus contains a convex torus with infinite boundary slope by assumption there exists a convex torus with boundary slope $-\frac{2}{6 k-1}$, which is critical. Thus we are left with two cases: $p_{1}=0, p_{2}=1, p_{3}=$ $3 k-1$, or $p_{1}=1, p_{2}=1, p_{3}=0$. 
Case 4. We finally assume that the basic slice $T_{2} \times\left[\frac{1}{2}, 1\right]$ has the same sign as $T_{3} \times I$. This covers the last two cases. Using a convex annulus between $T_{2} \times\left\{\frac{1}{2}\right\}$ and $T_{3} \times\left\{\frac{3 k-2}{3 k-1}\right\}$ that does not have a boundary parallel curves, we obtain a convex torus isotopic to $-\partial\left(M \backslash V_{1}\right)$ with boundary slope 1. By the Twisting Number Lemma with Legendrian slope $r_{1}=1$, we can increase the twisting number $n_{1}$ up to 1 . So by decreasing the twisting number $n_{1}$ we may assume that $T_{1} \times I$ and $T_{2} \times\left[\frac{1}{2}, 1\right]$ have the same sign. This assumption reduce this case to the previous case such as $p_{1}=p_{2}=0$ or $p_{1}=p_{2}=1$ which was proved to be overtwisted.

Now we are in a position to deal with the case where $A$ has no boundary-parallel dividing curve.

Proposition 2.7. If A has no boundary-parallel dividing curve, there exist, up to contact isotopy, at most $3 k-5$ tight contact structures on $M\left(-\frac{1}{2}, \frac{1}{3}, \frac{2}{6 k-1}\right)$.

Proof. Recall that the twisting numbers $n_{i}$ are $n_{1}=n_{2}=-2$ and $n_{3}=-1$. Thus the boundary slopes are

$$
s\left(-\partial\left(M \backslash V_{1}\right)\right)=\frac{-2}{-5}, \quad s\left(-\partial\left(M \backslash V_{2}\right)\right)=\frac{2}{-5}, \quad \text { and } s\left(-\partial\left(M \backslash V_{3}\right)\right)=\frac{1}{-3 k+1} .
$$

Applying the cut-and-round procedure to a convex annulus $A$ between $-\partial\left(M \backslash V_{1}\right)$ and $-\partial\left(M \backslash V_{2}\right)$, we obtain a torus with boundary slope $\frac{2}{5}-\frac{2}{5}+\frac{1}{5}=\frac{1}{5}$ on a torus $T$ isotopic to $\partial\left(M \backslash V_{3}\right)$. Since we have

$$
\left(\begin{array}{rc}
-1 & -3 k \\
2 & 6 k-1
\end{array}\right)\left(\begin{array}{r}
-5 \\
1
\end{array}\right)=\left(\begin{array}{c}
5-3 k \\
6 k-11
\end{array}\right)
$$

the convex torus isotopic to $\partial V_{3}$ has boundary slope

$$
-\frac{6 k-11}{3 k-5}=-2-\frac{1}{-(3 k-5)} \text {. }
$$

Thus it follows from the classification of the tight contact structures on a solid torus [Honda 2000, Theorem 2.3] that there exist, up to contact isotopy, exactly $|(-2+1)(3 k-5)|=3 k-5$ tight contact structures on a solid torus $V_{3}^{\prime \prime}$ of a singular fiber $f_{3}$ with boundary $T$. Since the boundary slopes of $\partial V_{1}$ and $\partial V_{2}$ are all $\frac{1}{-2}$, clearly there exists a unique tight contact structure on each $V_{1}$ and $V_{2}$. Moreover, the thickened torus obtained by the cut-and-round procedure above should be nonrotative for the manifold $M$ to be tight.

This completes the proof of Theorem 2.1.

\section{Proof of Corollary 1.2: lower bound}

We now construct $3 k-5$ holomorphically fillable contact structures on the Seifert fibered 3-manifold $M\left(-\frac{1}{2}, \frac{1}{3}, \frac{2}{6 k-1}\right)$. 
(a)

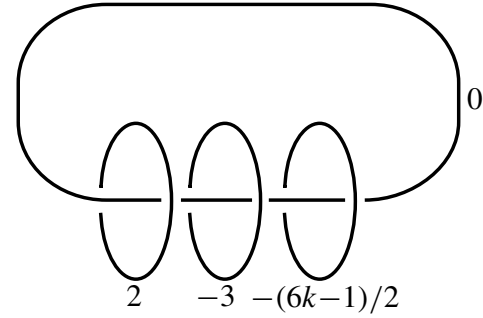

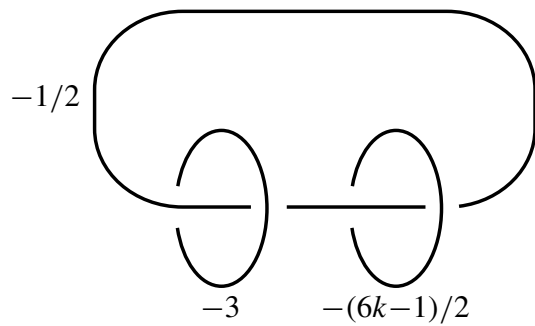

(b)

(c)

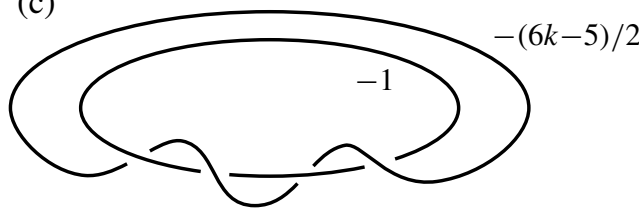

(e)

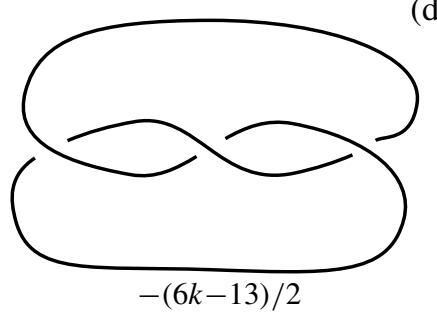

Figure 1. Surgery diagrams for $M\left(-\frac{1}{2}, \frac{1}{3}, \frac{2}{6 k-1}\right)$.

We use Kirby calculus to show that the manifold $M\left(-\frac{1}{2}, \frac{1}{3}, \frac{2}{6 k-1}\right)$ can be represented as Legendrian surgery on the framed link as follows. Indeed, the surgery description of the Seifert fibered 3-manifold $M\left(-\frac{1}{2}, \frac{1}{3}, \frac{2}{6 k-1}\right)$ is given in part (a) of Figure 1. Then we slam-dunk along the knot with framing coefficient 2 to obtain part (b). Then we perform the Rolfsen $(+2)$-twist along the knot with framing coefficient $-\frac{1}{2}$ to obtain (c). Next once more we do the Rolfsen $(+1)$-twist along the knot with framing coefficient -1 to obtain (d). Finally we do the inverse slamdunk to get (e). The last framed link can be realized as Legendrian links in several ways as shown in Figure 2 ( $l$ downward zig-zags plus one zig on the right and $m-l$ upward zig-zags on the left).

For any $k \geq 2$, we let $m=3 k-6$ and denote by $X_{k}^{l}(0 \leq l \leq m)$ the Stein manifolds with boundary constructed by attaching handles along these framed links. By applying [Lisca and Matić 1997, Theorem 2.1] we have $\left\langle\operatorname{PD}\left(c_{1}\left(X_{k}^{l}\right)\right), T\right\rangle=0$ and $\left\langle\operatorname{PD}\left(c_{1}\left(X_{k}^{l}\right)\right), S\right\rangle=2 l-m$. Thus by the same theorem or [Kronheimer and Mrowka 1997], we have at least $m+1=3 k-5$ nonisotopic holomorphically fillable contact structures on the Seifert fibered 3-manifold $M\left(-\frac{1}{2}, \frac{1}{3}, \frac{2}{6 k-1}\right)$. This complete the proof of Theorem 1.1. 


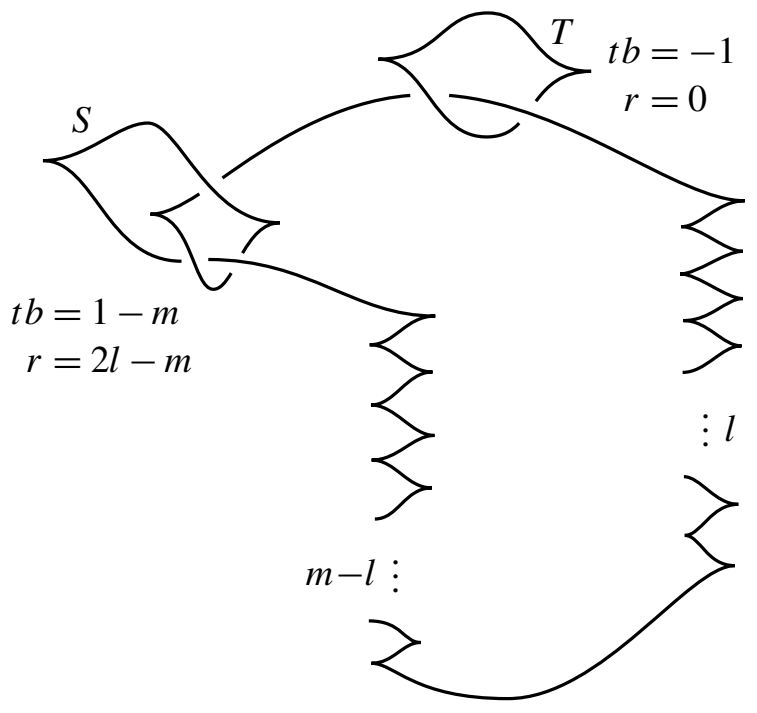

Figure 2. Legendrian surgery diagrams for $M\left(-\frac{1}{2}, \frac{1}{3}, \frac{2}{6 k-1}\right)$.

Remark. By [Lisca and Matić 1997, Proposition 2.2], the $3 k-5$ nonisotopic holomorphically fillable contact structures on the 3-manifold $M\left(-\frac{1}{2}, \frac{1}{3}, \frac{2}{6 k-1}\right)$ constructed in the proof of Theorem 1.1 are not homotopic as fields of 2-planes.

\section{Proof of Theorem 1.1}

The proof of Theorem 1.1 is closely modeled on that of Corollary 1.2, developed in Sections 2 and 3. Thus we just highlight the differences between the two proofs.

Throughout this section, $M$ with denote the Seifert 3-manifold $M\left(-\frac{1}{2}, \frac{1}{3}, \frac{\beta}{\alpha}\right)$ fibered over $S^{2}$ with three singular fibers $f_{i}$ with invariants $\left(-\frac{1}{2}, \frac{1}{3}, \frac{\beta}{\alpha}\right)$ satisfying $0<\frac{\beta}{\alpha}<\frac{1}{6}$, and we will keep the notations of the preceding two sections, unless stated otherwise. In this general case, the attaching maps $A_{1}$ and $A_{2}$ are the same as in Section 2, but now the attaching map $A_{3}: \partial V_{3} \rightarrow-\partial\left(S^{1} \times \Sigma\right)$ is given by

$$
A_{3}=\left(\begin{array}{rr}
\alpha & \alpha^{\prime} \\
-\beta & -\beta^{\prime}
\end{array}\right) \text {, }
$$

where $\alpha^{\prime} \beta-\alpha \beta^{\prime}=1$ with $0<\alpha^{\prime}<\alpha$ and $\alpha, \beta>0$. Note that $\beta^{\prime}>0$ and $\beta-\beta^{\prime}>0$. Then the boundary slope of $-\partial\left(M \backslash V_{3}\right)$ is

$$
-\frac{(\alpha-6 \beta) n_{2}-2 \beta}{\left(\alpha^{\prime}-6 \beta^{\prime}\right) n_{2}-2 \beta^{\prime}}
$$

Since $\frac{\beta}{\alpha}=\frac{\beta^{\prime}}{\alpha^{\prime}}+\frac{1}{\alpha \alpha^{\prime}}$ and $0<\frac{\beta}{\alpha}<\frac{1}{6}$, we see that $0<\frac{\beta^{\prime}}{\alpha^{\prime}}<\frac{1}{6}$. Hence the number $(4-1)$ is negative. 
Now, we work on a convex annulus between $-\partial\left(M \backslash V_{2}\right)$ and $-\partial\left(M \backslash V_{3}\right)$. Since $6 n_{2}+2<3 n_{2}+1 \leq 0$, we can apply the Imbalance Principle with $r_{3}$ equal to (4-1). Indeed, we first claim that we have

$$
0<\frac{\alpha^{\prime}-6 \beta^{\prime}}{\alpha-6 \beta}<1
$$

To see this, if the inequality (4-2) does not hold, we easily obtain using the relation $\alpha^{\prime} \beta-\alpha \beta^{\prime}=1$ that

$$
1 \geq(\alpha-6 \beta)\left(\beta-\beta^{\prime}\right),
$$

which implies that $\alpha-6 \beta=1$ and $\beta-\beta^{\prime}=1$. Thus

$$
1=\alpha^{\prime} \beta-\alpha \beta^{\prime}=\beta\left(\alpha^{\prime}-6 \beta+5\right)+1,
$$

which implies that $\beta=0$. This is a contradiction. Hence

$$
-2 \leq-1+\frac{1}{r_{3}} \leq-1-\frac{\alpha^{\prime}-4 \beta^{\prime}}{\alpha-4 \beta} .
$$

Note also that the fraction on the right lies between 0 and 1 . Thus by the Twisting Number Lemma as in Lemma 2.2 with Legendrian ruling slope $r_{3}$ equal to the equation (4-1), we have

$$
n_{3} \leq-1+\frac{1}{r_{3}}<-1
$$

as long as $n_{2} \leq-2$. This implies that we increase the twisting number by one as long as the twisting number $n_{3}$ is less than or equal to -2 . Now it is easy to see that the rest of the proof in Lemma 2.2 works without any modifications. Hence we conclude that we can increase the twisting numbers $n_{1}, n_{2}$, and $n_{3}$ up to -2 , -2 , and -1 , respectively.

Now assume that the convex annulus $A$ as in Lemma 2.4 has a boundary-parallel dividing curve. Since we do not have any change for the gluing maps $A_{1}$ and $A_{2}$, we can increase the twisting numbers $n_{1}$ and $n_{2}$ up to 0 and -1 as in Lemma 2.4. Furthermore, since the slope of $\partial V_{3}$ corresponding to the slope 0 (resp. $\infty$ ) on $\partial\left(M \backslash V_{3}\right)$ is $-\frac{\beta}{\beta^{\prime}}$ (resp. $\left.-\frac{\alpha}{\alpha^{\prime}}\right)$ and $-\frac{\beta}{\beta^{\prime}}<-\frac{\alpha}{\alpha^{\prime}}<0$ using $\alpha^{\prime} \beta-\alpha \beta^{\prime}=1$, we have a Legendrian regular fiber with twisting number 0 .

Next as in Proposition 2.5, we show that there does not exist any tight contact structures on $M$, up to isotopy, which have the twisting numbers $n_{1}=0, n_{2}=-1$, and $n_{3}=-1$. But this can be shown in the similar way as in Section 2, since $M \backslash \bigcup_{i=1}^{3} V_{i}$ is diffeomorphic to $S^{1} \times \Sigma$ with boundary slope $0,-\frac{1}{2}$, and

$$
-\frac{1}{3}<-\frac{\beta-\beta^{\prime}}{\alpha-\alpha^{\prime}}=-\left(\frac{\beta}{\alpha}+\frac{1}{\alpha\left(\alpha-\alpha^{\prime}\right)}\right)<0
$$


To be precise, it follows from the inequality (4-4) that there exists a convex standard neighborhood $V_{3}^{\prime \prime}$ of the singular fiber $f_{3}$ containing $V_{3}$ inside $V_{3}^{\prime}$ whose boundary slope $s\left(-\partial\left(M \backslash V_{3}^{\prime \prime}\right)\right)$ is $-\frac{1}{3}$. Let $T_{3}^{\prime \prime}$ denote the convex torus bounding $V_{3}^{\prime \prime}$. Thus we have a thickened torus $T_{3}^{\prime \prime} \times I$ with $T_{3}^{\prime \prime} \times\{0\}=-\partial\left(M \backslash V_{3}^{\prime \prime}\right)$ and $T_{3}^{\prime \prime} \times\{1\}=-\partial\left(M \backslash V_{3}^{\prime}\right)$ whose boundary slopes are $-\frac{1}{3}$ and $\infty$. Note also that the thickened torus $T_{3}^{\prime \prime} \times I$ is the union of three basic slices $T_{3}^{\prime \prime} \times\left[\frac{i}{3}, \frac{i+1}{3}\right]$ with boundary slopes $-\frac{1}{3-i}$ and $-\frac{1}{2-i}$ for $i=0,1,2$ which is a continued fraction block.

As before, let $p_{i}$ denote the number of positive basic slices in $T_{i} \times I(i=1,2)$ and let $p_{3}$ be the number of positive basic slices in $T_{3}^{\prime \prime} \times I$. Then there exist at least $2 \times 3 \times 3=18$ possible tight contact structures on $M \backslash\left(V_{1} \cup V_{2} \cup V_{3}^{\prime \prime}\right)$. But we will show that these cases do not occur under the assumption that there exists a Legendrian regular fiber with twisting number 0 . This will clearly finish the proof that there does not exist any tight contact structures on $M$, up to isotopy, which have the twisting numbers $n_{1}=0, n_{2}=-1$, and $n_{3}=-1$. The proof is just a repetition of the arguments in Section 2. But in our case the critical slopes are respectively

$$
s\left(-\partial\left(M \backslash \tilde{V}_{1}\right)\right)=\frac{1}{2}, \quad s\left(-\partial\left(M \backslash \tilde{V}_{2}\right)\right)=-\frac{1}{3}, \quad \text { and } s\left(-\partial\left(M \backslash \tilde{V}_{3}\right)\right)=-\frac{\beta}{\alpha}>-\frac{1}{6} .
$$

Using an argument similar to that of Case 1 of Proposition 2.5, we are left with the cases $p_{2}=0$ and $p_{3}=2$ or $3, p_{1}=1$ and $p_{3}=0$ or 3 , and $p_{2}=1$ and $p_{3}=0$ or 1 . Next as in Case 2 we are left with the following six cases $p_{1}=0=p_{2}$ and $p_{3}=1$, $p_{1}=0, p_{2}=1$ and $p_{3}=3, p_{1}=1, p_{2}=0$ and $p_{3}=2, p_{1}=p_{2}=1$ and $p_{3}=0$, and $p_{1}=1, p_{2}=2$, and $p_{3}=0$ or 1 . Applying the argument in Case 3, we can reduce the above six cases to three cases $p_{0}=0, p_{2}=1$ and $p_{3}=3, p_{1}=1, p_{2}=1$ and $p_{3}=0$, and $p_{1}=1, p_{2}=0$ and $p_{3}=2$. This is possible, since $-\frac{1}{5}<-\frac{\beta}{\alpha}$ and the thickened torus obtained by cut-and-round procedure along the convex annulus between $-\partial\left(M \backslash V_{1}^{\prime \prime}\right)$ and $-\partial\left(M \backslash V_{2}^{\prime \prime}\right)$ as in Case 3 in the proof of Proposition 2.5 contains a convex torus with infinite boundary slope. Finally, an argument as in Case 4 concludes that there does not exist any tight contact structures on $M$, up to isotopy, which have the twisting numbers $n_{1}=0, n_{2}=-1$, and $n_{3}=-1$.

We are thus left with the case that $A$ has no boundary-parallel dividing curve. In this case, it follows as in Proposition 2.7 that we have the convex torus isotopic to $\partial V_{3}$ with boundary slope $-(\alpha-5 \beta) /\left(\alpha^{\prime}-5 \beta^{\prime}\right)$ which is equal to

$$
\left[r_{l}, r_{l-1}, \ldots, r_{0}+5\right] \text {. }
$$

In fact, we can see this as follows. Since $\left(\alpha^{\prime}-5 \beta^{\prime}\right) \beta-(\alpha-5 \beta) \beta^{\prime}=1$, the fraction $-(\alpha-5 \beta) / \beta$ is written as $\left[5+r_{0}, r_{1}, \ldots, r_{l}\right]$. Thus the fraction

$$
-\frac{\alpha-5 \beta}{\alpha^{\prime}-5 \beta^{\prime}}
$$


is equal to $\left[r_{l}, r_{l-1}, \ldots, r_{0}+5\right]$; see [Ghiggini et al. 2004, Lemma 2.5], for example. Hence we conclude that there exist at most $\left|r_{0}+5\right|\left|r_{1}+1\right| \cdots\left|r_{l}+1\right|$ tight contact structures on $M$ up to isotopy by the classification of Honda.

Finally we need to construct $\left|r_{0}+5\right|\left|r_{1}+1\right| \cdots\left|r_{l}+1\right|$ tight contact structures on $M$ by the Legendrian surgery argument. But $M$ is the result of a surgery on the right-handed trefoil knot with surgery coefficient $-\frac{\alpha}{\beta}+6<0$. Hence, as in Section 3 or as in [Gompf 1991], we can show that there exist at least

$$
\left|r_{0}+5\right|\left|r_{1}+1\right| \cdots\left|r_{l}+1\right|
$$

tight contact structures on $M$. This completes the proof of Theorem 1.1.

\section{Acknowledgements}

The author is very grateful to an anonymous reader for carefully reading an earlier version of this paper and helping the author improve it to the present form. He also acknowledges the support of Korea Institute of Advanced Study (KIAS) as an associate member during the work.

\section{References}

[Colin et al. 2003] V. Colin, E. Giroux, and K. Honda, "On the coarse classification of tight contact structures", pp. 109-120 in Topology and geometry of manifolds (Athens, GA, 2001), edited by G. Matic and C. McCrory, Proc. Symp. Pure Math. 71, Amer. Math. Soc., Providence, RI, 2003. MR 2005a:53146 Zbl 1052.57036

[Eliashberg 1990] Y. Eliashberg, "Topological characterization of Stein manifolds of dimension > 2", Internat. J. Math. 1:1 (1990), 29-46. MR 91k:32012 Zbl 0699.58002

[Etnyre and Honda 2001] J. B. Etnyre and K. Honda, "On the nonexistence of tight contact structures”, Ann. of Math. (2) 153:3 (2001), 749-766. MR 2002d:53119 Zbl 1061.53062

[Ghiggini and Schönenberger 2003] P. Ghiggini and S. Schönenberger, "On the classification of tight contact structures", pp. 121-151 in Topology and geometry of manifolds (Athens, GA, 2001), Proc. Symp. Pure Math. 71, Amer. Math. Soc., Providence, RI, 2003. MR 2004m:53146 Zbl 1045.57013

[Ghiggini et al. 2004] P. Ghiggini, P. Lisca, and A. I. Stipsicz, "Classification of tight contact structures on small Seifert 3-manifolds with $e_{0} \geq 0$ ", preprint, 2004. math.SG/0406080

[Gompf 1991] R. E. Gompf, "Nuclei of elliptic surfaces”, Topology 30:3 (1991), 479-511. MR 92f: 57042 Zbl 0732.57010

[Gompf 1998] R. E. Gompf, "Handlebody construction of Stein surfaces", Ann. of Math. (2) 148:2 (1998), 619-693. MR 2000a:57070 Zbl 0919.57012

[Honda 2000] K. Honda, "On the classification of tight contact structures. I", Geom. Topol. 4 (2000), 309-368 (electronic). MR 2001i:53148 Zbl 0980.57010

[Kronheimer and Mrowka 1997] P. B. Kronheimer and T. S. Mrowka, "Monopoles and contact structures", Invent. Math. 130:2 (1997), 209-255. MR 98h:57058 Zbl 0892.53015

[Lisca and Matić 1997] P. Lisca and G. Matić, "Tight contact structures and Seiberg-Witten invariants", Invent. Math. 129:3 (1997), 509-525. MR 98f:57055 Zbl 0882.57008 
[Wu 2004] H. Wu, "Tight Contact Small Seifert Spaces with $e_{0} \neq 0,-1,-2$ ", preprint, 2004. math.GT/0402167

Received October 9, 2003. Revised October 18, 2004.

JINHONG KIM

DEPARTMENT OF MATHEMATICS

KAIST, KUSONG-DONG, YUSONG-GU

DAEJON 305-701

SOUTH KOREA

jinkim11@kaist.ac.kr 